\title{
UN DOMAINE CONSIDÉRABLE
}

\author{
H. DECENCIĖRE-FERRANDIĖRE
}

Class. Oxford $681: 922.1$

Le régime forestier que l'Office national des forêts est dorénavant chargé de mettre en œuvre, et qui est défini par le livre II du Code forestier, s'applique aux forêts et terrains à bolser appartenant, en totalité ou en indivis, à l'Etat, ainsi qu'aux forêts susceptibles d'aménagement, d'exploitations régulières ou de reconstitution, et aux terrains à boiser propriété des départements, des communes, des sections de communes, des établissements publics et d'utilitè publique, des sociétés de secours mutuel et des caisses d'épargne, ou sur lesquels ces collectivités ou personnes morales ont des droits de propriété indivis.

Au $1^{\text {er }}$ janvier 1970, la surface totale du domaine soumis à ce régime en France métropolitaine était de $4084684 \mathrm{ha}$, dont :

- 1655954 ha de forêts domaniales constituant le domaine forestier privé de l'Etat. Cet ensemble comprend d'une part les forêts dont la gestion et l'équipement ont été confiés à l'Office, soit $1444745 \mathrm{ha}$, et d'autre part celles dans lesquelles il n'est légalement chargé que de l'application du régime forestier, soit 215209 ha.

- 11434 ha boisés appartenant également à l'Etat, et soumis au régime forestier, mais affectés à divers services, notamment à la défense nationale.

- 13197 ha de foréts départementales.

- 2351639 ha de forêts communales et sectionales.

- 41284 ha de forêts d'établissements publics.

-2203 ha de forêts d'établissements d'utilité publique et de sociétés de secours mutuel.

- 8973 ha de foréts de caisses d'épargne.

Dans les départements d'Outre-mer le domaine soumis au régime forestler couvre 93953 ha à la Réunion, 11899 ha à la Martinique, 38210 ha à la Guadeloupe, 8045000 ha à la Guyane.

Au $1^{\text {er }}$ janvier 1877, date à laquelle fut établie la première statistique forestière complète, la surface soumise en France métropolitaine n'était que de 3198690 ha compte tenu de l'Alsace-Lorraine, dont 1137012 ha domaniaux, 2033937 ha communaux et sectionaux et 27741 ha appartenant à des établissements publics. II n'y avait alors ni forêts départementales, ni forêts d'établissements d'utilité publique, de sociétés de secours mutuel ou de calsses d'épargne.

Du $1^{\text {er }}$ janvier 1877 au $1^{\text {er }}$ janvier 1970 , solt en un peu moins de cent ans, la surface soumise s'est accrue de 885994 ha, soit de $27,5 \%$ dont 518942 ha pour le domaine forestier privé de l'Etat $(45,7 \%)$ et 317702 ha pour les forêts communales et sectionales $(15,6 \%)$. 
L'augmentation de la surface des forêts domaniales est essentiellement due à l'acquisition par l'Etat :

- soit de terrains situés en zone montagneuse, à l'intérieur des périmètres de lutte contre l'érosion (372000 ha),

- soit de forêts privées ruinées à restaurer ou de terrains à boiser (147000 ha).

Celle des forêts communales et sectionales trouve son origine d'une part dans une meilleure application du régime forestier instauré en 1827, et dont la mise en œuvre souleva à l'origine des oppositions de la part de certaines collectivités, et d'autre part dans la mise en valeur forestière de vacants communaux abandonnés par le pâturage ou la culture.

L'importance du domaine soumis au régime forestier est très variable d'une région à l'autre. Le tableau ci-après indique, par direction régionale de l'Office national des forêts, la surface soumise et ce qu'elle représente par rapport à la surface totale et à la surface boisée de la région correspondante

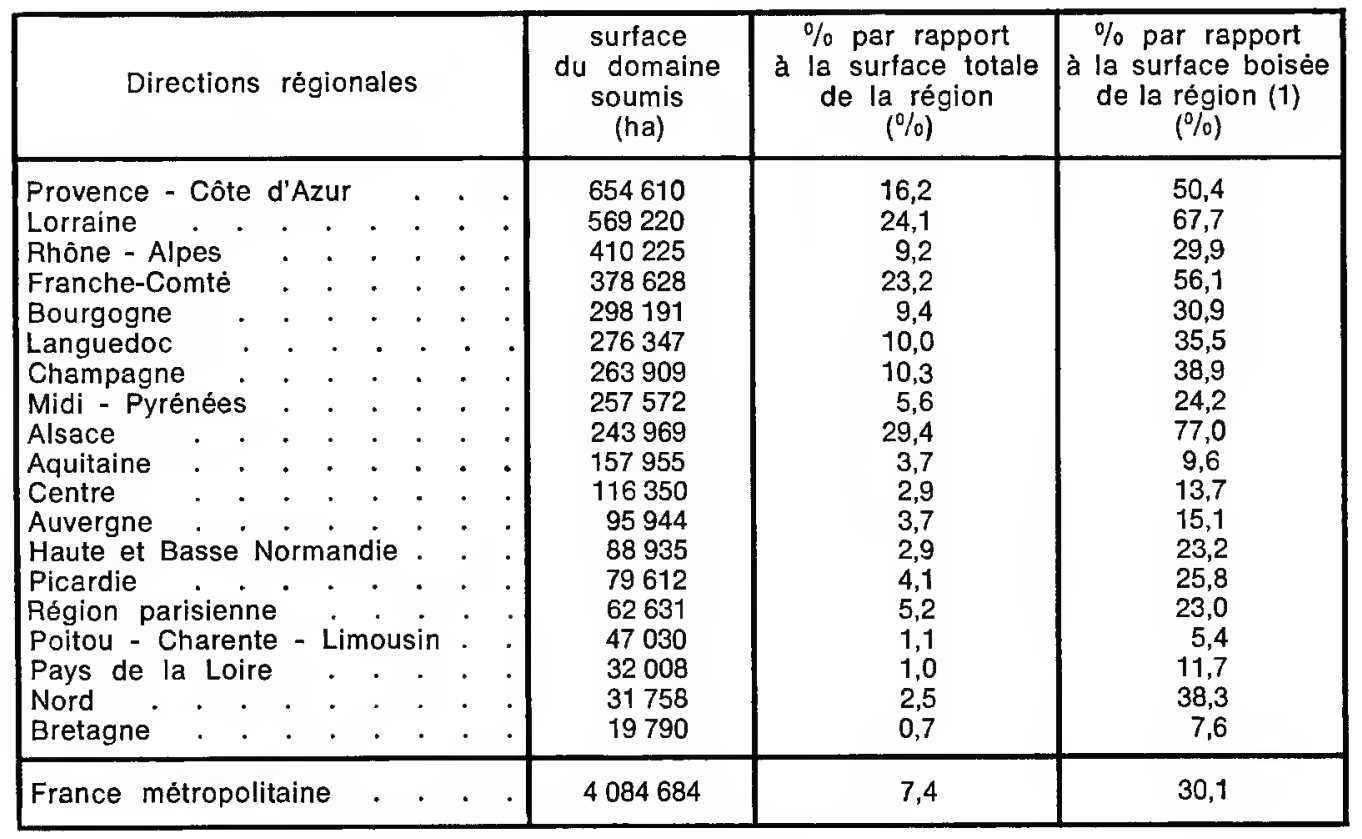

(1) D'après des chiffres fournis par l'Inventaire forestier.

De ces chiffres il résulte une très grande difterence dans la répartitlon des forêts soumises dont l'importance varie de $80 \%$ de la surface boisée en Alsace à $5 \%$ seulement en PoitouCharente-Limousin. Si l'on considère d'autre part l'importance de ce domaine par rapport à la surface totale de chaque région on constate qu'en Alsace il représente près de $30 \%$ de celle-ci et près du quart en Lorraine et en Franche-Comté. C'est dire l'importance du rôle de l'Office dans ces régions, non seulement au seul plan forestier, mais également sur celui de l'économie générale et de l'aménagement du territoire.

Dans l'ouest par contre le domaine soumis est peu important. II convient cependant de signaler que depuis quelques dizaines d'annèes un effort a été fait pour améliorer cette situation. 
C'est ainsi que de 1914 à 1970, alors que la surface totale du domaine soumis augmentait de $15 \%$ sur l'ensemble du territoire, elle s'est trouvée majorée de $53 \%$ en Bretagne, de $30 \%$ en Poitou-Charente-Limousin, de $35 \%$ en Aquitaine, de $27 \%$ dans les Pays de la Loire et de $21 \%$ en Haute et Basse Normandie. Cet accroissement de surface est dû à des achats de forêts privées par l'Etat, surtout en Normandie, en Bretagne et dans les Pays de la Loire, et à des soumissions de terrains sectionaux dans le Limousin et communaux en Aquitaine. Le domaine soumis se compose d'éléments de natures très différentes, qui vont de la futaie pleine de chêne de tranchage ou de résineux de haute qualité, aux terrains nus, difficilement de chene... PMOTO CMAIGNEAUD

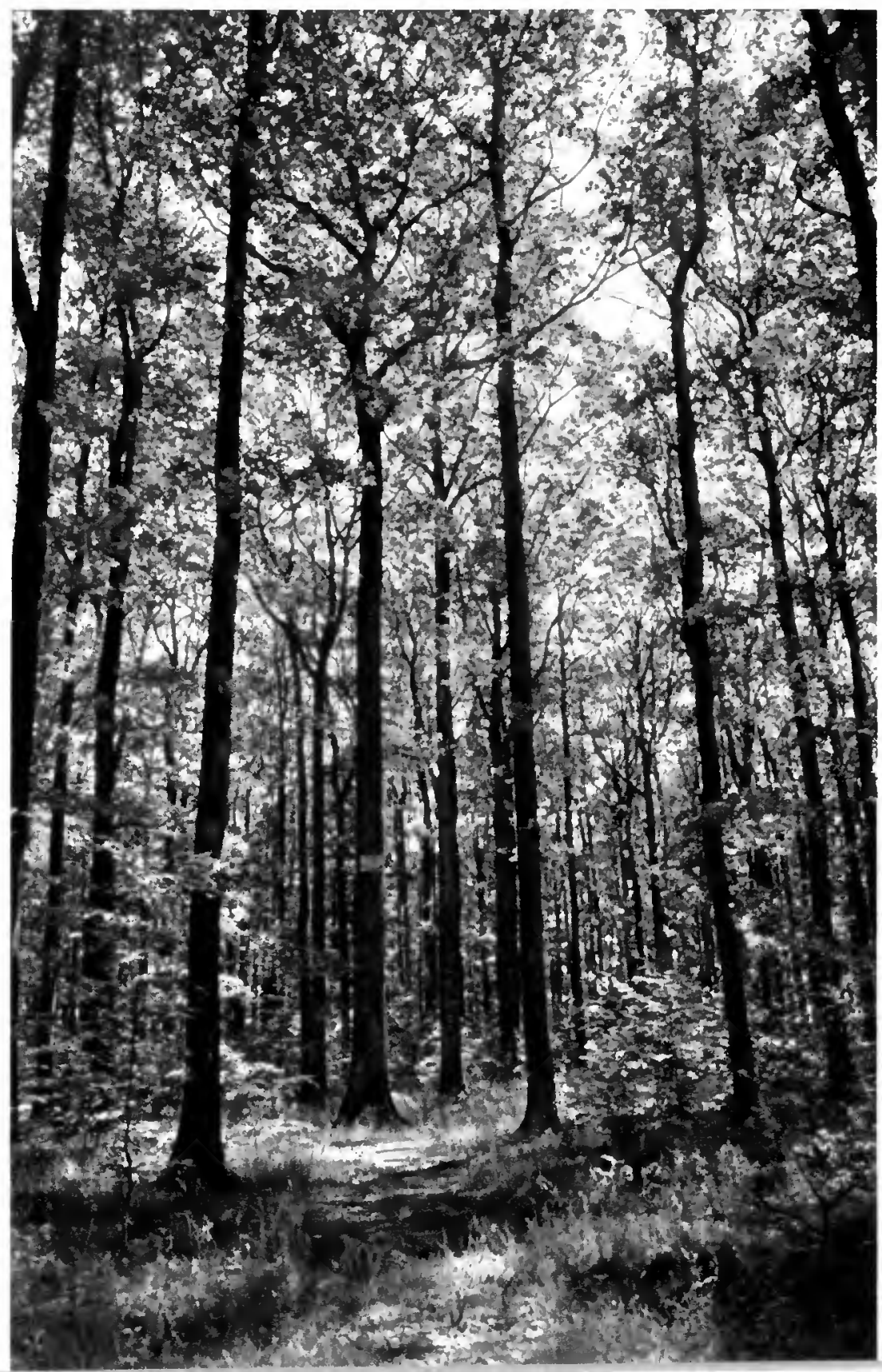




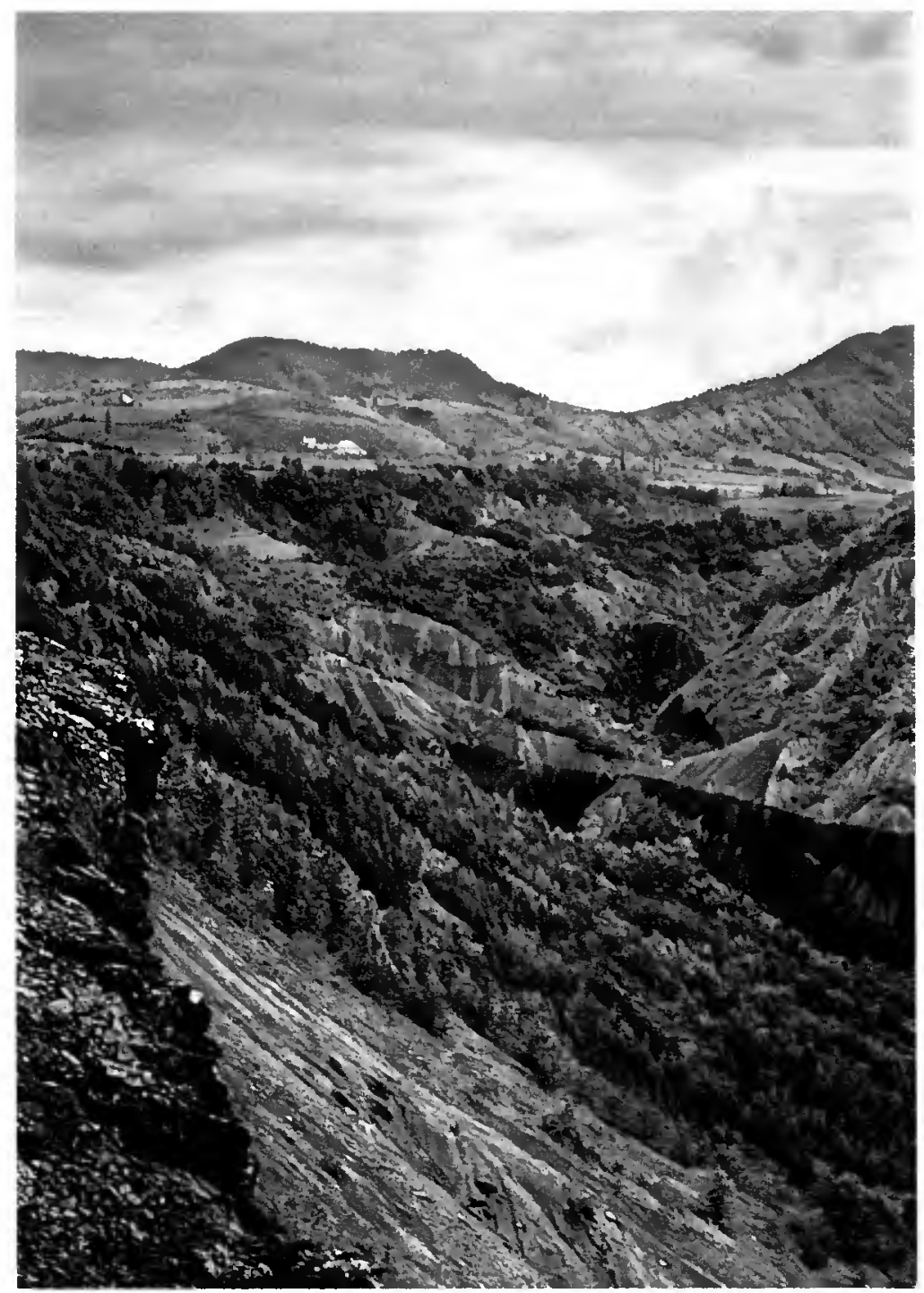

... eux terrelns nus difflcllement boisables ou méme non boisables PHOTO BOUTIN

boisables ou même non boisables, en raison de leur altitude. Cette diffèrence tient bien entendu à la nature et à la situation des terrains soumis mais aussi, et beaucoup, aux traitements qui ont été appliqués à ces peuplements, qu'il s'agisse d'anciennes forêts royales traitées depuis fort longtemps pour la production de bois de qualité (de bois de marine notamment aux $17^{\circ}$ et $18^{\circ}$ siècles), de massifs destinès à l'affouage ou frappés de servitudes usagères et restés jusqu'à maintenant à l'état de taillis simple ou de taillis-sous-futaie, d'anciens taillis ayant autrefois alimentés les forges ou les salines et convertis plus ou moins complètement en futaies depuis le Second empire, de terrains nus acquis en montagne dans le cadre de la lutte contre l'érosion et boisés en résineux ou simplement regazonnés, de plantations de pin maritime constituées au $19^{\mathrm{e}}$ siècle sur les dunes de la côte aquitaine, de boisements réalisẻs sur des terrains abandonnés par le pâturage ou rendus incultivables par les grands combats de la guerre 1914-1918, de forêts privées ruinées par des coupes abusives ou des incendies et acquises par l'Etat, ou certaines collectivités, en vue de leur restauration ou même 
de forēts en bon état achetées à titre de placements par des caisses d'épargne ou des sociétès mutualistes.

En fin de compte, à travers le domaine soumis, on perçoit toute l'histoire de la forêt française et l'immense effort de toutes les génèrations de forestiers qui se sont appliquées à l'améliorer et à l'étendre.

L'Office national des forêts auquel il est maintenant confié, entend bien poursuivre cette tâche et notamment mettre au point et proposer à l'Etat une politique dynamique d'achat de forêts ou de terrains à boiser. A ce propos, il convient de signaler qu'actuellement d'importantes acquisitions sont déjà en cours de réalisation, notamment dans la Région parisienne, le département du Nord, le massif de la Sainte-Baume, le Plateau de Millevaches, l'Aveyronnais et que la plus grande forêt privée française, celle d'Arc-en-Barrois en Haute-Marne (10700 ha) a été intégrée au domaine forestier privé de l'Etat le 15 janvier dernier.

\section{Henri DECENCIÈRE-FERRANDIËRE}

Ingénieur en chef du G.R.E.F.

Chef du département juridique et immabilier de

$$
\text { I'O.N.F. }
$$

4, avenue de Saint-Mandé

PARIS - 120 